\title{
Mathematical Model of Two-Degree-of-Freedom Direct Drive Induction Motor Considering Coupling Effect
}

\author{
Jikai Si ${ }^{\dagger}$, Lujia Xie*, Junbo Han*, Haichao Feng*, Wenping Cao** and Yihua Hu*** \begin{abstract}
rotary and helical motion, has a wide application in special industry such as industrial robot arms. It is inevitable that the linear motion and rotary motion generate coupling effect on each other on account of the high integration. The analysis of this effect has great significance in the research of two-degreeof-freedom motors, which is also crucial to realize precision control of them. The coupling factor considering the coupling effect is proposed and addressed by 3D finite element method. Then the corrected mathematical model is presented by importing the coupling factor. The results from it are verified by $3 \mathrm{D}$ finite element model and prototype test, which validates the corrected mathematical model.
\end{abstract} \\ Abstract - The two-degree-of-freedom direct drive induction motor, which is capable of linear,
}

Keywords: Coupling effect, Mathematical model, 3-D finite element method, Two-degree-offreedom direct-drive induction motor

\section{Introduction}

Two degrees of freedom motors capable of linear, rotary or helical motion have the advantages of simple structure, small abrasion, high reliability and low maintenance cost. A further applicative area opening to such machines is the vehicular ambit, such as servo-actuators for gearboxes or robot ambit [1-4].

Bolognesi presented a dual-stator linear-rotary permanent motor [5]. The motor consists of two identical stators and a PM mover. The coupling effect between linear and rotary motion can be neglected. An improved type of linear-rotary permanent motor was introduced [6]. A twodirectional d-q transformation was proposed to decouple the inter-relationship between linear and rotary motion [7]. However, the linear-rotary permanent motors structures are mechanically complicated and the entire control system needs to be highly sophisticated [8]. The characteristics of a two-degree-of-freedom motor - rotary-linear induction motor with twin-armature were analyzed [9]. The linear motion generates dynamic end effect on both linear and rotary armature. Reference [10] adopted the combination of transient time-domain finite element model and frequencydomain slip frequency technique to model the dynamic end effect and analyzed the influence of the linear motion on

$\dagger$ Corresponding Author: School of Electrical Engineering and Automation, Henan Polytechnic University, China.

(sijikai527@126.com)

* School of Electrical Engineering and Automation, Henan Polytechnic University, China. (xielujia1993@163.com, jess_han@126.com, fhc@hpu.edu.cn)

** School of Electronics, Electrical Engineering and Computer Science, Aston University, U.K. (w.p.cao@aston.ac.uk)

*** Department of Electrical Engineering and Electronics, University of Liverpool, U.K. (Y.Hu35@liverpool.ac.uk)

Received: July 19, 2016; Accepted: March 22, 2017 rotary motion. A rotating linear switched reluctance motor presented in [11], is to integrate rotating motion and linear motion based on the minimum reluctance principle. However, the coupling of this motor has occurred when both rotating motion and linear motion are produced. A method of decoupling control for the reduction of coupling effect of the motor's operation has been implenmented. Reference [12] presented an optimized two degrees of freedom switched reluctance motor and it was applied to the electric vessel. Two PID controllers and position tracking controllers were employed for linear and rotary motions to power the linear and rotary part in some sorted order, which reduced coupling effect of the motor's operation by decoupling control.

Although certain types of helical-motion motors have been developed over the years [13-17], many areas require further research. For example, modeling coupling effects is a significant challenge as it requires a solution considering the two degrees of mechanical freedom in the motion. In this paper, a novel two-degree-of-freedom direct drive induction motor (2-DoFDDIM) was analyzed [18-20]. A 2-D analytic model and a 3-D finite element model of the motor were established to analyze the magnetic coupling and motion coupling between rotary part and linear part. A novel mathematical model of 2-DoFDDIM was proposed to consider the coupling effects by importing coupling factor. And it was then verified by 3-D finite element model simulation and prototype test results.

\section{Structure and Principles of Operation}

The structure of 2-DoFDDIM is shown in Fig. 1. The motor consists of a rotary motion arc-shape stator, a linear 
motion arc-shape stator and a mover. The mover consists of iron cylinder covered with a thin copper layer. When the rotary stator is energized, rotating magnetic field will be generated. According to electromagnetic induction principal, the voltage and current will be induced on the rotor surface and then produce electromagnetic torque. In the similar way, the traveling wave magnetic field will be generated when the linear stator is powered and the force will follow. If only rotary or linear stator is energized, the motor does single degree mechanical motion. When both of them are energized, the motor produces helical motion. Thus, the motor can produce rotary, linear and helical motion. The main parameters of the motor are shown in Table 1.

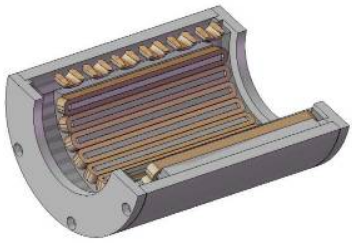

(a)

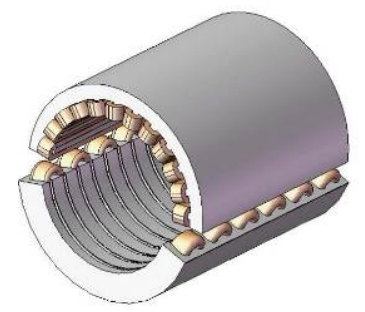

(c)

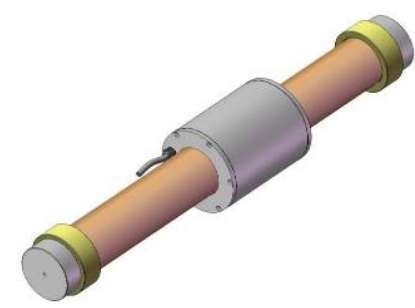

(e)

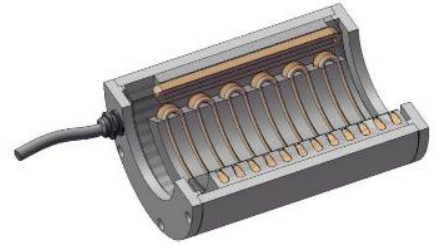

(b)

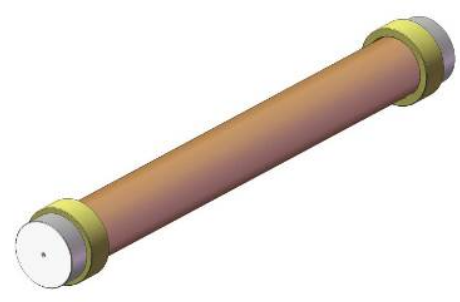

(d)

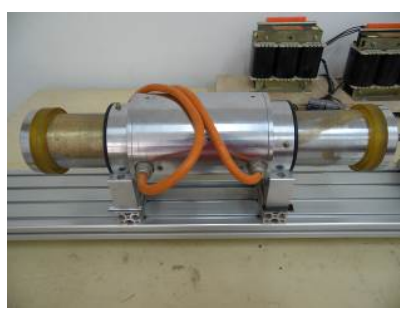

(f)
Fig. 1. Structure of 2DoFDDIM: (a) Rotary motion arcshape stator; (b) Linear motion arc-shape stator; (c) Integrated stator; (d) Mover; (e) Assembly of 2DoFDDIM. (f) Prototype of 2DoFDDIM

Table 1. The main structure parameters of the 2-DoFDDIM

\begin{tabular}{c|c|c}
\hline \multirow{2}{*}{ Item } & \multicolumn{2}{|c}{ Value/Dimension } \\
\cline { 2 - 3 } & Rotary Part & Linear Part \\
\hline Rated power (PN) & $1.1 \mathrm{~kW}$ & $1.1 \mathrm{~kW}$ \\
Rated voltage (VN) & $220 \mathrm{~V}(\mathrm{Y})$ & $220 \mathrm{~V}(\mathrm{Y})$ \\
Frequency (f) & $50 \mathrm{~Hz}$ & $5 \mathrm{~Hz}$ \\
Pole pairs (p) & 2 & 2 \\
Stator inner diameter & $98 \mathrm{~mm}$ & $98 \mathrm{~mm}$ \\
Stator outer diameter & $155 \mathrm{~mm}$ & $155 \mathrm{~mm}$ \\
Stator axial length & $130 \mathrm{~mm}$ & $156 \mathrm{~mm}$ \\
Air-gap length & $1 \mathrm{~mm}$ & $1 \mathrm{~mm}$ \\
Slot number & 12 & 12 \\
\hline
\end{tabular}

\section{Coupling Effect}

On account of the coupling effect, the motor will produce unwanted linear directional force upon the movement of rotating motion, and vice versa. For instance, the $127 \mathrm{~V}-50 \mathrm{~Hz}$ power source is used to power the rotary windings, whereas the linear windings are under different cases: powered or not powered. The mover rotary speeds are reported in Fig. 2.

As shown in Fig. 2, when the rotary windings are powered $127 \mathrm{~V}-50 \mathrm{~Hz}$ power source and the linear windings are not powered, the mover rotary speed is $766.67 \mathrm{r} / \mathrm{min}$. However, when the linear windings are powered with $127 \mathrm{~V}-5 \mathrm{~Hz}$ power source, the mover rotary speed reduces to 550r/min. This phenomenon, caused by coupling effect, appears when linear windings are powered, and it is speculated that the traveling magnetic field restrains the rotary motion of mover.

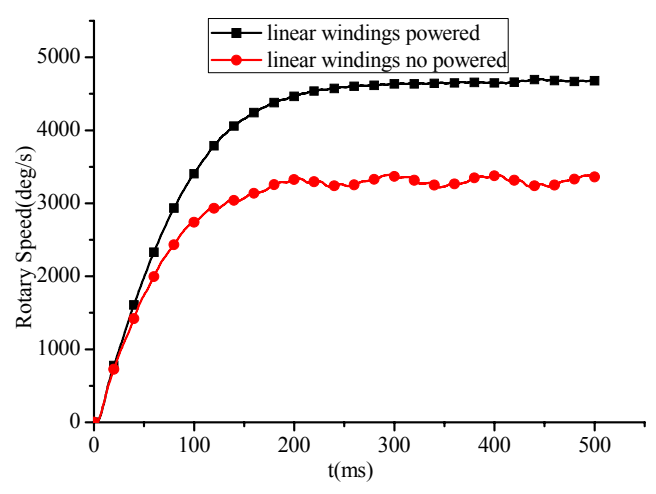

Fig. 2. The rotary speed

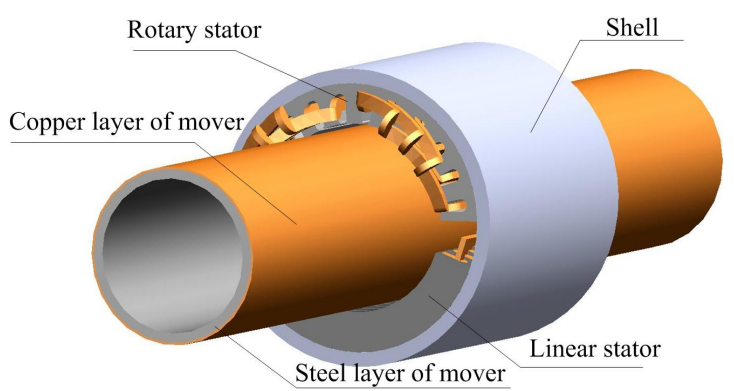

Fig. 3. The model of 2-DoFDDIM

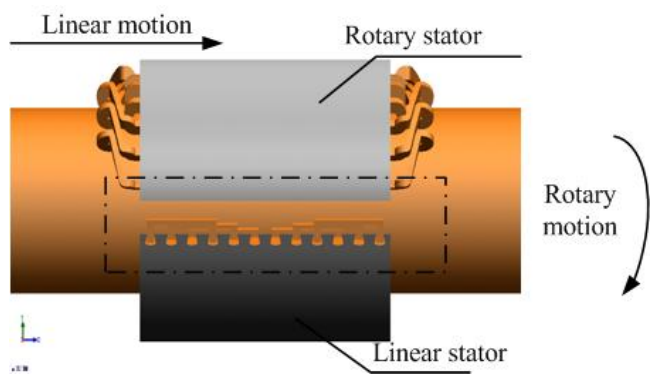

Fig. 4. The right side view of 2-DoFDDIM without shell 


\subsection{Analysis and model}

Fig. 3 shows the model of 2-DoFDDIM and the right side view without shell is shown in Fig. 4.

When both of rotary and linear windings are powered, the rotary stator produces rotating magnetic field and the linear stator produces traveling magnetic field. When the mover makes a rotary motion, the conductive layer of the mover will cut the traveling magnetic field at the end of linear stator shown in the dashed box in Fig. 4. When it moves in or out of the traveling magnetic field, there is a force that opposes motion and then the eddy current is induced. The conductive layer can be reckoned as the composition of countless conducting bars. In order to calculate the resistent force, it is assumed that there is one conducting bar in the conductive layer with length is $L$. The conducting bar speed is $v$ and the traveling magnetic flux density is $B$. Then the induction potential is $e$. The Eq. (1) can be obtained:

$$
e=B L v
$$

The electric magnetic force of the conducting bar $F_{1}$ is

$$
F_{1}=B I L=\frac{B^{2} L^{2}}{R} v
$$

where $R$ is the resistance of the conductive bar.

Thus, the force of the conductive layer $F$ can be derived.

$$
F=\sum_{\mathrm{i}=1}^{\mathrm{K}} F_{1}
$$

Where $K$ is the number of the conductive bars at the eddy current place and the value of it is unknown.

It can be obtained from (2) and (3) that

$$
F=K \frac{B^{2} L^{2}}{R} v=k v
$$

where $k=K \frac{B^{2} L^{2}}{R}$.

The (4) shows that the resistant force $F$ is proportional to the speed of conductive layer. Considering the rotary motion, it can be concluded that the resistant torque caused by traveling magnetic field is proportional to the rotary speed of mover.

$$
T_{1}=k_{s} n
$$

where $k_{s}$ is the rotary coupling factor. $n$ is the rotary speed of mover. $T_{1}$ is the resistant torque.

Then the rotary part electromagnetic torque should be corrected as shown in Eq. (6).

$$
T=T_{e}-T_{1}=T_{e}-k_{s} n
$$

$T_{e}$ is the rotary part electromagnetic torque when the coupling effect is not considered.

Similarly, the rotating magnetic field also will restrain linear motion. So the linear electromagnetic thrust equation $F_{e}$ should be corrected as shown in Eq. (7).

$$
F=F_{e}-F_{1}=F_{e}-k_{l} v
$$

$F_{e}$ is the linear electromagnetic thrust when the coupling effect is not considered. $k_{l}$ is the linear coupling factor. $v$ is the linear speed of mover.

\subsection{3-D finite element calculation}

The particular structure and multi-form of motion of 2DoFDDIM make it difficult to calculate the coupling factors using analytic method. Thus the 3-D finite element method (FEM) is used to calculate the coupling factors indirectly.

Fig. 5 (a) gives the 3-D finite element model of the 2DoFDDIM, which is established in the finite-element software Magnet. Fig. 5 (b) gives the flux density distribution when both the rotary and linear windings are powered.

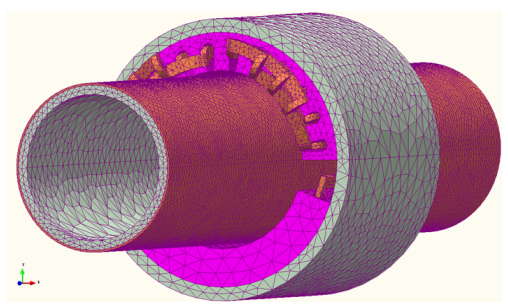

(a) 3-D finite element model of 2-DoFDDIM

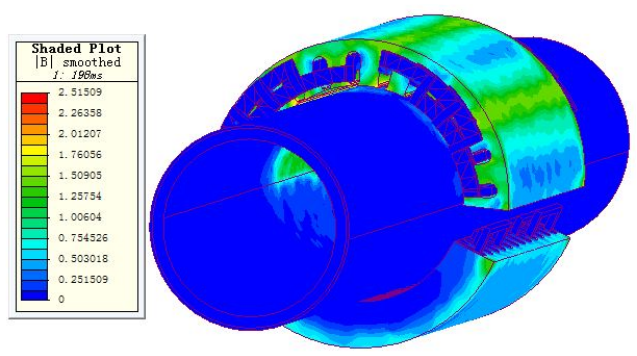

(b) Flux density distribution of 2-DoFDDIM

Fig. 5. Finite element analysis of 2-DoFDDIM

Table 2. Rotary speed of mover under different situation

\begin{tabular}{cccc}
\hline \multirow{2}{*}{$\begin{array}{c}\text { Rotary } \\
\text { voltage }(\mathrm{V})\end{array}$} & $\begin{array}{c}\text { Rotary } \\
\text { Frequency } \\
(\mathrm{Hz})\end{array}$ & $\begin{array}{c}\text { Rinear } \\
\text { windings } \\
\text { powered }\end{array}$ & $\begin{array}{c}\text { Linear windings } \\
\text { not powered }\end{array}$ \\
\hline 76.2 & 30 & 450 & 319.67 \\
88.9 & 35 & 525 & 380 \\
101.6 & 40 & 600 & 444.33 \\
114.3 & 45 & 675 & 510.83 \\
127 & 50 & 7500 & 589 \\
\hline
\end{tabular}


Table 3. Resistant torque caused by traveling magnetic field

\begin{tabular}{c|c|c|c}
\hline $\begin{array}{c}\text { Rotary } \\
\text { voltage }(\mathrm{V})\end{array}$ & $\begin{array}{c}\text { Rotary frequency } \\
(\mathrm{Hz})\end{array}$ & $\begin{array}{c}\text { Rotary given } \\
\text { speed }(\mathrm{r} / \mathrm{min})\end{array}$ & $\begin{array}{c}\text { Resistant } \\
\text { torque }(\mathrm{Nm})\end{array}$ \\
\hline 76.2 & 30 & 319.67 & 2.886 \\
88.9 & 35 & 380 & 3.58 \\
101.6 & 40 & 444.33 & 4.257 \\
114.3 & 45 & 510.83 & 4.748 \\
127 & 50 & 3534 & 5.18 \\
\hline
\end{tabular}

Table 4. $k_{n}$ under different rotary speeds

\begin{tabular}{c|c|c|c|c|c}
\hline $\begin{array}{c}\text { Rotary speed } \\
(\mathrm{r} / \mathrm{min})\end{array}$ & 319.67 & 380 & 444.33 & 510.83 & 589 \\
\hline$k_{n}$ & 0.0015 & 0.00157 & 0.0016 & 0.00155 & 0.00147 \\
\hline
\end{tabular}

The rotary windings are powered with a constant proportion of voltage to frequency power source, while the linear windings are under two situations: powered with $127 \mathrm{~V} 10 \mathrm{~Hz}$ power source or without power. The rotary speeds of mover are shown in Table 2.

As shown in Table 2, whether the linear windings are powered or not has a significant influence on rotary speed. The result shows that the traveling magnetic field indeed restrains the rotary motion of mover.

On the premise that linear windings are not powered, the mover rotary speeds are set as the mover rotary speeds when linear windings are powered. Then the load torque is calculated. In fact, the load torque is equal to the resistant torque caused by traveling magnetic field. The results of FEM are shown in Table 3.

As shown in Table 3, the resistant torque increases with the increase of the rotary speed. In order to verify the Eq. (5), resistant torque is divided by the corresponding rotary speed, as shown in Table 4.

$$
k_{n}=\frac{T_{n}}{v_{n}} n=1,2,3,4,5
$$

As shown in Table $4, k_{n}$ under different rotary speeds is close to others, which verifies the Eq. (5). The average of $k_{n}$ is derived.

$$
k_{s}=\frac{\sum_{n=1}^{5} k_{n}}{5}=0.001537
$$

So $k_{s}$ is the rotary coupling factor when linear windings are powered $127 \mathrm{~V}-10 \mathrm{~Hz}$. Then the linear coupling factor $k_{l}$ is derived by adopting the similarly method, $k_{l}=11.897$.

\section{Mathematical Model of 2-DoFDDIM}

Because of the linear and rotary part are powered with different power sources, the motor can be divided into two

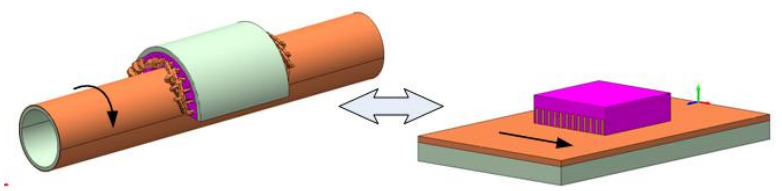

(a)

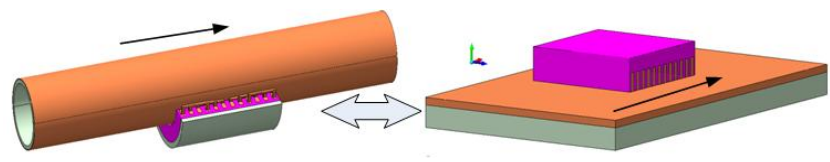

(b)

Fig. 6. Equivalent transformation of rotary and linear part: (a) Equivalent transformation of rotary part; (b) Equivalent transformation of linear part

parts: rotary arc-shape LIM and linear arc-shape LIM. As shown in Fig. 6, the rotary arc-shape LIM and the linear arc-shape LIM can be equivalently transferred to flat LIM. Thus, the mathematical models of rotary and linear part can be built based on the mathematical model of LIM respectively. The coupling effects can be considered in torque equation and thrust equation.

\subsection{Mathematical model of rotary part}

1) The voltage equation of rotary part

$$
\left\{\begin{array}{l}
u_{1 \alpha}=R_{1} i_{1 \alpha}+R_{2} f\left(Q_{1}\right)\left(i_{1 \alpha}+i_{2 \alpha}\right)+\frac{d \psi_{1 \alpha}}{d t} \\
u_{1 \beta}=R_{1} i_{1 \beta}+R_{2} f\left(Q_{1}\right)\left(i_{1 \beta}+i_{2 \beta}\right)+\frac{d \psi_{1 \beta}}{d t} \\
0=R_{2} i_{2 \alpha}+R_{2} f\left(Q_{1}\right)\left(i_{1 \alpha}+i_{2 \alpha}\right)+\frac{d \psi_{2 \alpha}}{d t}+\omega_{1} \psi_{2 \alpha} \\
0=R_{2} i_{2 \beta}+R_{2} f\left(Q_{1}\right)\left(i_{1 \beta}+i_{2 \beta}\right)+\frac{d \psi_{2 \beta}}{d t}-\omega_{1} \psi_{2 \beta}
\end{array}\right.
$$

where $\left(u_{1 \alpha}, u_{1 \beta}\right),\left(i_{1 \alpha}, i_{1 \beta}\right),\left(\psi_{1 d}, \psi_{1 q}\right)$ and $R_{1}$ are the $\alpha \beta$ axis voltages, currents, flux linkages and resistance of the rotary part stator. $\left(i_{2 d}, i_{2 q}\right),\left(\psi_{2 \alpha}, \psi_{2 \beta}\right)$ and $R_{2}$ are the $\alpha \beta$-axis currents, flux linkages and resistance of the rotary part rotor. $\omega_{1}$ denotes the rotor angular speed. $f\left(Q_{1}\right)$ is used to consider the rotary part end effect [11]. Where $f\left(Q_{1}\right)=\frac{1-e^{-Q_{1}}}{Q_{1}} Q_{1}=\frac{D_{1} R_{2} \pi}{L_{2} \omega_{1} \tau_{1}}$. The $D_{1}$ denotes the length of rotary stator. $\tau_{1}$ is the pole pitch of the rotary part.

2) The flux linkage equation of rotary part

$$
\left\{\begin{array}{l}
\psi_{1 \alpha}=\left(L_{1}-L_{s m} f\left(Q_{1}\right)\right) i_{1 \alpha}+L_{s m}\left(1-f\left(Q_{1}\right)\right) i_{2 \alpha} \\
\psi_{1 \beta}=\left(L_{1}-L_{s m} f\left(Q_{1}\right)\right) i_{1 \beta}+L_{s m}\left(1-f\left(Q_{1}\right)\right) i_{2 \beta} \\
\psi_{2 \alpha}=\left(L_{2}-L_{s m} f\left(Q_{1}\right)\right) i_{2 \alpha}+L_{s m}\left(1-f\left(Q_{1}\right)\right) i_{1 \alpha} \\
\psi_{2 \beta}=\left(L_{2}-L_{s m} f\left(Q_{1}\right)\right) i_{2 \beta}+L_{s m}\left(1-f\left(Q_{1}\right)\right) i_{1 \beta}
\end{array}\right.
$$


where $L_{1}, L_{s m}, L_{2}$ are the $\alpha \beta$-axis stator self inductance, mutual inductance and rotor self inductance.

Due to the coupling effects, the rotary part torque equation has to be corrected. The revised torque equation from (6) can be obtained in (12):

$$
T=n_{p 1} \frac{L_{s m}\left(1-f\left(Q_{1}\right)\right)}{\left(L_{2}-L_{s m} f\left(Q_{1}\right)\right)}\left(\psi_{1 \alpha} i_{1 \beta}-\psi_{1 \beta} i_{1 \alpha}\right)-k_{s} n
$$

where $n$ is the rotary speed of mover, $n_{p 1}$ is the number of rotary part pole-pairs

\subsection{Mathematical model of linear part}

1) The voltage equation of linear part

$$
\left\{\begin{array}{l}
u_{3 \alpha}=R_{3} i_{3 \alpha}+R_{4} f\left(Q_{1}\right)\left(i_{3 \alpha}+i_{4 \alpha}\right)+\frac{d \psi_{3 \alpha}}{d t} \\
u_{3 \beta}=R_{3} i_{3 \beta}+R_{4} f\left(Q_{1}\right)\left(i_{3 \beta}+i_{4 \beta}\right)+\frac{d \psi_{3 \beta}}{d t} \\
0=R_{4} i_{4 \alpha}+R_{4} f\left(Q_{1}\right)\left(i_{3 \alpha}+i_{4 \alpha}\right)+\frac{d \psi_{4 \alpha}}{d t}+\omega_{2} \psi_{4 \alpha} \\
0=R_{4} i_{4 \beta}+R_{4} f\left(Q_{1}\right)\left(i_{3 \beta}+i_{4 \beta}\right)+\frac{d \psi_{4 \beta}}{d t}-\omega_{2} \psi_{4 \beta}
\end{array}\right.
$$

where $\left(u_{3 \alpha}, u_{3 \beta}\right),\left(i_{3 \alpha}, i_{3 \beta}\right),\left(\psi_{3 \alpha}, \psi_{3 \beta}\right)$ and $R_{3}$ are the $\alpha \beta$ axis voltages, currents, flux linkages and resistance of the linear part primary. $\left(i_{4 \alpha}, i_{4 \beta}\right),\left(\psi_{4 \alpha}, \psi_{4 \beta}\right)$ and $R_{4}$ are the $\alpha \beta$-axis currents, flux linkages and resistance of the linear part secondary. $\omega_{2}$ denotes the secondary angular speed. $f\left(Q_{2}\right)$ is used for considering the linear part end effect. where $\omega_{2}=\frac{\pi}{\tau_{2}} v_{2}, f\left(Q_{2}\right)=\frac{1-e^{-Q_{2}}}{Q_{2}}, Q_{2}=\frac{D_{2} R_{4}}{L_{4} v_{2}}$. The $D_{2}$ denotes the length of primary stator. $\tau_{2}$ is the pole pitch of the linear part. $v_{2}$ is the secondary speed.

2) The flux linkage equation of linear part

$$
\left\{\begin{array}{l}
\psi_{3 \alpha}=\left(L_{3}-L_{l m} f\left(Q_{2}\right)\right) i_{3 \alpha}+L_{l m}\left(1-f\left(Q_{2}\right)\right) i_{4 \alpha} \\
\psi_{3 \beta}=\left(L_{3}-L_{l m} f\left(Q_{2}\right)\right) i_{3 \beta}+L_{l m}\left(1-f\left(Q_{2}\right)\right) i_{4 \beta} \\
\psi_{4 \alpha}=\left(L_{4}-L_{l m} f\left(Q_{2}\right)\right) i_{4 \alpha}+L_{l m}\left(1-f\left(Q_{2}\right)\right) i_{3 \alpha} \\
\psi_{4 \beta}=\left(L_{4}-L_{l m} f\left(Q_{2}\right)\right) i_{4 \beta}+L_{l m}\left(1-f\left(Q_{2}\right)\right) i_{3 \beta}
\end{array}\right.
$$

where $L_{3}, L_{l m}, L_{4}$ are the $\alpha \beta$-axis primary self inductance, mutual inductance and secondary self inductance.

Due to the coupling effect, the linear part thrust equation has to be corrected. The revised thrust equation from (7) can be obtained in (15).

$$
F=n_{p 2} \frac{\pi L_{l m}\left(1-f\left(Q_{2}\right)\right)}{\tau_{2}\left(L_{4}-L_{l m} f\left(Q_{2}\right)\right)}\left(\psi_{3 \alpha} i_{3 \beta}-\psi_{3 \beta} i_{3 \alpha}\right)-k_{l} v
$$

where $v$ is the linear speed of mover, $n_{p 2}$ is the number of linear part pole-pairs.

\section{Comparison Results}

The simulating results of the corrected mathematical model are verified by FEM and measurements carried out on the prototype of 2-DoFDDIM shown in Fig. 7.

In order to verify the mathematical model of rotary part, the rotary windings are powered with $127 \mathrm{~V}-50 \mathrm{~Hz}$ power source, while the linear windings are not powered. It can be known that the traveling magnetic field does not exist, thus, the rotary coupling factor $k_{s}=0$. The rotary speeds of FEM, mathematical model and test are shown in Fig. 8.

As shown in Fig. 8, the results of mathematical model coincide with the one of FEM well and the difference between mathematical model and test is less than $13 \%$, which meets accuracy requirements. The reasons of errors are the ignorance of mechanical loss, wind resistance and bearing friction in mathematical model and FEM.

In order to verify the mathematical model of linear part, the linear windings are powered with $127 \mathrm{~V}-10 \mathrm{~Hz}$ power source, while the rotary windings are not powered. The rotating magnetic field does not exist. $k_{l}=0$. The rotary speeds of FEM, mathematical model and test are shown in Fig. 9.

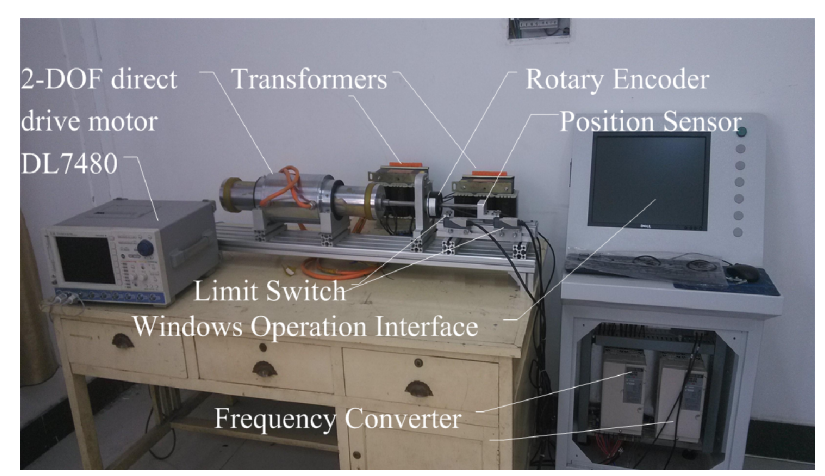

Fig. 7. Prototype testing platform system of 2-DoFDDIM

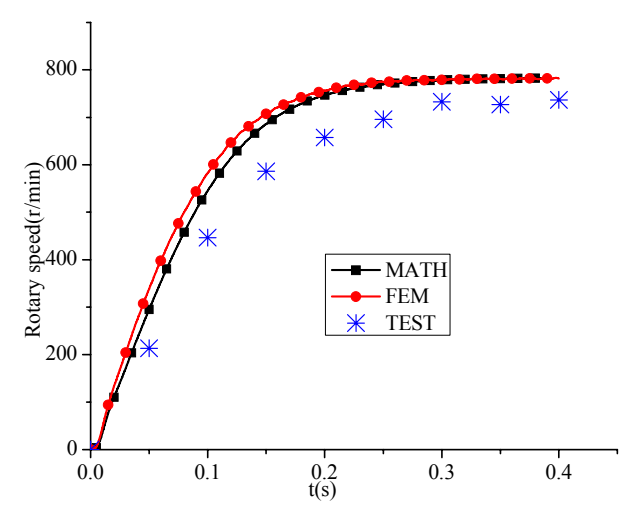

Fig. 8. Rotary speed 


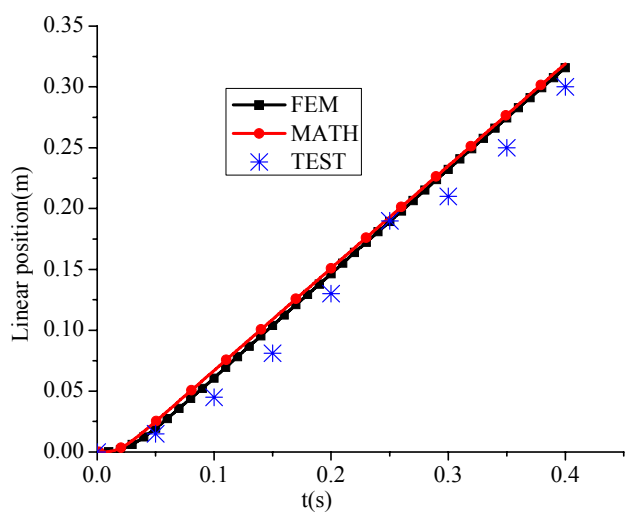

Fig. 9. Linear position

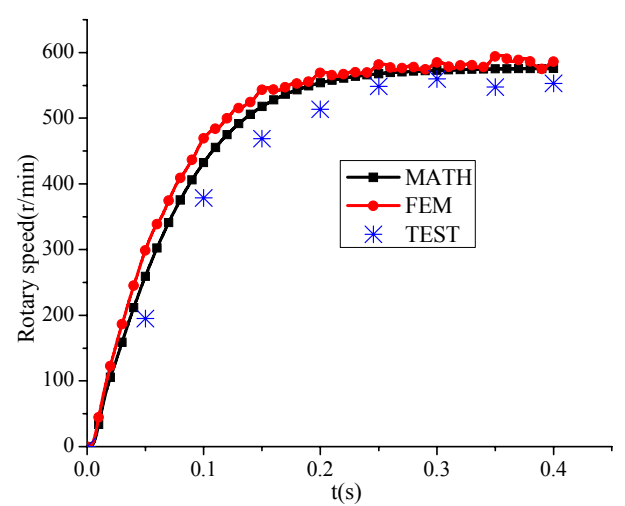

(a)

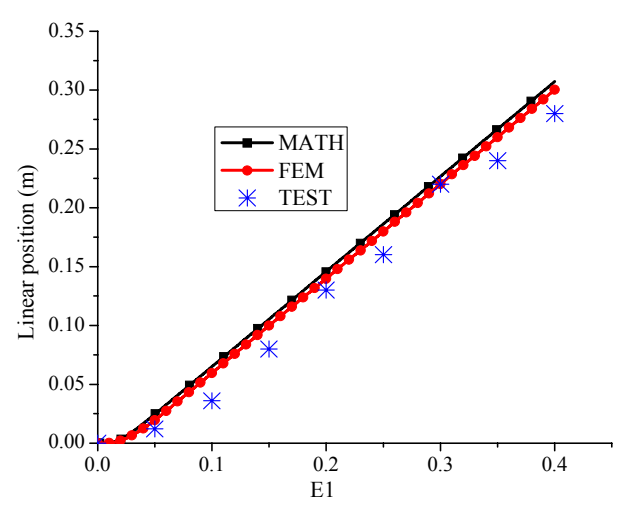

(b)

Fig. 10. Rotary speed and linear position of mathematical model, FEM and test: (a) Rotary speed; (b) Linear position

As shown in Fig. 9, at $0.4 \mathrm{~s}$, the linear positions of mathematical model, FEM and test are $0.3195 \mathrm{~m}, 0.315 \mathrm{~m}$ and $0.31 \mathrm{~m}$, respectively. The error is less than $2.9 \%$, which verify the mathematical model of linear part.

The coupling effects must be considered when both of rotary and linear windings are powered. To verify the coupling mathematical model, the rotary windings and linear coil are powered with $127 \mathrm{~V}-50 \mathrm{~Hz}$ and $127 \mathrm{~V}-10 \mathrm{~Hz}$ power sources. $k_{s}=0.001537, k_{l}=12$. The results of mathematical model, FEM and test are shown in Fig. 10.
Compared Fig. 8 with Fig. 10 (a) and Fig. 9 with Fig. 10 (b), it can be seen that the steady rotary speed decreases $24 \%$ and linear position reduces $6.7 \%$ at $0.4 \mathrm{~s}$ on account of the coupling effect, which is represented by $k_{s}$ and $k_{l}$ in mathematical model. It is worth noting that the reduction of linear position is less than rotary speed. The main reason of it is that the linear resistant force caused by coupling effect is directly proportional to linear speed, as shown in formulas (5)-(7). For 2-DoFDDIM, the linear speed is smaller than rotary speed.

As shown in Fig.10 (a), the steady rotary speed of mathematical model, FEM and test are $590.5 \mathrm{r} / \mathrm{min}$, $586 \mathrm{r} / \mathrm{min}$ and $552.86 \mathrm{r} / \mathrm{min}$. The error is less than $6.8 \%$. As shown in Fig.10 (b), the linear position of mathematical model, FEM and test are $0.31 \mathrm{~m}, 0.30 \mathrm{~m}$ and $0.28 \mathrm{~m}$ at $0.4 \mathrm{~s}$. The error is less than $9.8 \%$. The results of mathematical model are consistent with that of FEM and test, which verify the mathematical model considering the coupling effects between linear and rotary part.

\section{Conclusion}

This paper analyzed the coupling effect between linear and rotary part of 2-DoFDDIM. The electromechanical performances of the motor are affected by the coupling effects. There will be resistant torque (or thrust) when the motor produces both two degrees of mechanical freedom in the motion, which is proportional to rotary speed (or linear speed) of the mover. The rotary coupling factor and linear coupling factor are calculated by 3-D FEM. A corrected mathematical model of 2-DoFDDIM considering the coupling effects was established. The results obtained from the test carried out on the experimental model do not differ much from the ones got from 3-D finite element model and mathematical model simulation. Thus, they validate the proposed methodology and coupling effect, and it will be wider used in the control of 2DoFDDIM as well as the other multi-degree-of- freedom motors.

\section{Acknowledgements}

This works is supported by National Natural Science Foundation of China under grant 51277054, atural Science Foundation of Henan province 162300410117, Henan Polytechnic University Innovation Team T2015-2.

\section{References}

[1] E M. "Rotary-Linear Induction Motor with Solid Rotor," Rozpr. Electrotech, 1976, 2:383-408.

[12] Bolognesi P, Bruno O, Taponecco L. "Dual-Function 
Wheel Drives Using Rotary-Linear Actuators in Electric and Hybrid Vehicles," 35th Annual Conference of IEEE Industrial Electronics, IECON, Porto, 2009. 3916-3921.

[3] Turner, K. Ramsay, R. Clark and D. Howe, "DirectDrive Rotary-Linear Electromechanical Actuation System for Control of Gearshifts in Automated Transmissions," 2007 IEEE Vehicle Power and Propulsion Conference, Arlington, TX, 2007, pp. 267272.

[4] Y. Fujimoto, T. Suenaga, Y. Wakayama, K. Sawai and A. Z. Shukor, "Force control of a spiral motor and its application to musculoskeletal biped robot," The 12th IEEE International Workshop on Advanced Motion Control, Sarajevo, 2012, pp. 1-6.

[5] P. Bolognesi and V. Biagini, "Modeling and control of a rotary-linear drive using a novel isotropic brushless machine," XIX International Conference on Electrical Machines, Rome, 2010, pp. 1-6.

[6] P. Jin, S. H. Fang, H. Lin Z.Q. Zhu and Y. K. Huang, "Analytical Magnetic Field Analysis and Prediction of Cogging Force and Torque of a Linear and Rotary Permanent Magnet Actuator," IEEE Transactions on Magnetics, Oct. 2011, 47, (10), pp. 3004-3007.

[7] P. Jin, H. Y. Lin, S. H. Fang and S.L. Ho, "Decoupling Control of Linear and Rotary Permanent Magnet Actuator Using Two-Directional d-q Transformation," IEEE Transactions on Magnetics, Oct. 2012, 48, (10), pp. 3004-3007.

[8] J. F. Pan, Z. Yu, and N. C. Cheung, "Performance Analysis and Decoupling Control of an Integrated Rotary-Linear Machine With Coupled Magnetic Paths," IEEE Transactions on Magnetics, Feb. 2014, 50, (10), pp. 761-764.

[9] Ebrahim Amiri, Pavani Gottipati and Ernest A. Mendrela. "3-D Space Modeling of Rotary-Linear Induction Motor with Twin-Armature," IEEE International Conference on Electric Machines \& Drives, Newport Beach, CA, 2011, pp.202-206.

[10] Ebrahim Amiri, Mariusz Jagiela, Oleksandr Dobzhanski and Ernest Mendrela, "Modeling Dynamic End Effects in Rotary Armature of Rotary-Linear Induction Motor," IEEE International Conference on Electric Machines \& Drives, Chicago, IL, 2013, pp. 1088-1091.

[11] Ioana B, Lorand S, Mircea R. "A Novel RotaryLinear Switched Reluctance Motor," Journal of Computer Science and Control Systems, 2012, 1(5): 13-16.

[12] Li S Y, Cheng K W. "A New Two-degree of Freedom Switched Reluctance Motor for Electric Vessel," 6th International Conference on Power Electronics Systems and Applications (PESA), Hong Kong, 2015. 1-6

[13] S. Yasukazu, "Development of 2-Degree-of-Freedom rotational/linear switched reluctance motor," IEEE
Trans. Magnetics., vol. 43, no. 6, pp. 2564-2566, Jun. 2007.

[14] Peyman Yadmellat, Alexander S. Shafer, and Mehrdad R. Kermani. "Design and development of a single-motor, two-dOF, safe manipulator," IEEE/ ASME Transactions on Mechatronics, vol. 19, no. 4, Augest, 2014.

[15] Chiao-Hua Cheng, Shao-Kang Hung, "A piezoelectric 2-degree-of-freedom nano-stepping motor with parallel design," IEEE/ASME Transactions on Mechatronics, vol. 21, no. 4, pp. 2197-2199, Aug. 2016.

[16] M. Tsukano, Y. Sakaidani, K. Hirata, N. Niguchi, S. Maeda and A. Zaini, "Analysis of 2-degree of freedom outer rotor spherical actuator employing 3-D finite element method," IEEE Trans. on Magnetics., vol. 49, no. 5, pp. 2233-2236, May, 2013.

[17] K. J. Meessen, J. J. H.Paulides and E. A. Lomonova. "Analysis of novel magnetization pattern for 2-dof rotary-linear actuators," IEEE Trans.on Magnetics., vol. 48, no. 11, pp. 3867-3870, Nov. 2012.

[18] Jikai Si, Haichao Feng, Liwang $\mathrm{Ai}$, Yihua $\mathrm{Hu}$ and Wenping Cao. "Design and Analysis of a 2-dof splitstator induction motor," IEEE Trans. Energy Conversion, 2015, 30, (3), pp.1200-1208.

[19] Si Ji-kai, Si Meng, Feng Hai-chao and Xu Xiao-zhuo, "Research Status and Development of Two-Degreeof-Freedom Direct Drive Motor," Transactions of China Electrotechnical Society, Feb. 2013, 28, (2), pp.97-107.

[20] Si Jikai, Ai Liwang, Feng Haichao, Zhu Yifeng and $\mathrm{Hu}$ Yihua. "Analysis on coupling effect of 2-DOF direct drive induction motor based on 3-D model," 17th International Conference on Electrical Machines and Systems, ICEMS 2014, Hangzhou, 2014, pp. 1157-1163.

[21] G Kang and K. Nam, "Field-oriented control scheme for linear induction motor with the end effect". IEEE Proceedings-Electric Power Applications, Nov. 2005, 152, (6), pp. 1565-1572.

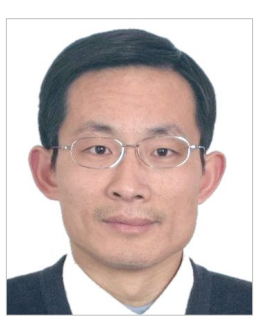

Jikai Si was born in Henan Province, China, in 1973. He received the B.Eng. and M. Eng. degrees from Jiaozuo Institute and Technology, Henan Polytechnic University, Jiaozuo, China, in 1998 and 2005, respectively. He received the $\mathrm{Ph} . \mathrm{D}$. degree in The School of Information and Electrical Engineering at China University of Mining and Technology, Xuzhou, China. Currently, he is a Professor in the Henan Polytechnic University. His main research interests include the theory, application and control of special motor. 


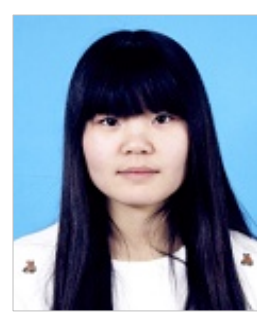

Lujia Xie was born in Henan Province, China, in 1995. She received bachelor's degree from Henan Polytechnic University, Jiaozuo, China, in 2014. Now she is attending graduate school in School of Electrical Engineering and Automation, Henan Polytechnic University, China. Her main research interests include the optimization design of linear and rotary machines the theory and application of special motor.

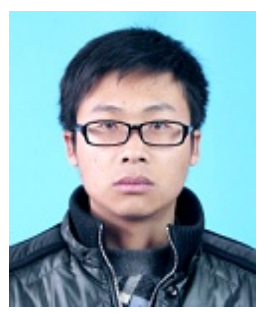

Junbo Han was born in Henan Province, China, in 1990. He received bachelor's degree from Henan Polytechnic University, Jiaozuo, China, in 2013. Now he is attending graduate school in School of Electrical Engineering and Automation, Henan Polytechnic University, China. His main research interests include the theory and control of special motor.

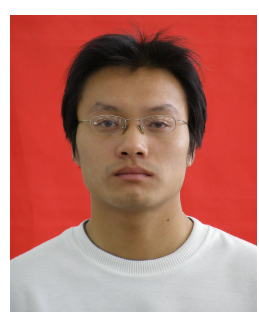

Haichao Feng was born in China in 1983, and received the B.S. and M.S. degrees in electrical engineering and automation, control theory and control engineering from School of Electrical Engineering and Automation, Henan Polytechnic University, china, in 2005 and 2008, respectively. His research interests are the optimization design of linear and rotary machines, power electronics, and their controls.

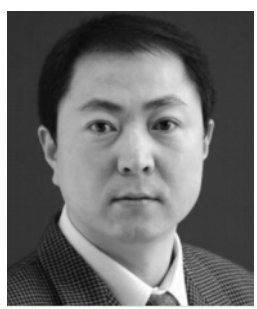

Wenping Cao (M'05-SM'11) received the B.Eng. degree in electrical engineering from Beijing Jiao-tong University, China, in 1991, and the Ph.D. degree in electrical machines and drives from the University of Nottingham, U.K., in 2004. He is currently a professor with Aston University, U.K. His research interests include thermal performance of electric machines, drives and power electronics.

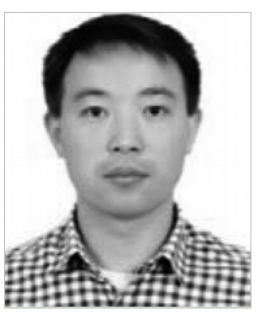

Yihua Hu (M'13) received the B.S. degree in elec-trical motor drives and the $\mathrm{Ph} . \mathrm{D}$. degree in power electronics and drives, both from China University of Mining and Technology, Jiangsu, China, in 2003 and 2011, respectively. Between 2011 and 2013, he was with the College of Electrical Engineering,

Zhejiang University as a Postdoctoral Fellow. Between November 2012 and February 2013, he was an academic Visiting Scholar with the School of Electrical and Electronic Engi-neering, Newcastle University, Newcastle upon Tyne, U.K. He is currently a Senior Lecturer with University of Liverpool, U.K. His research interests include PV generation systems, dc-dc/dc-ac converters, and electrical motor drives. 